Revue internationale P.M.E.

Économie et gestion de la petite et moyenne entreprise

Revue

internationale

PME

\title{
Très petite entreprise et marketing : les causes d'un malentendu
}

\section{Jean-Claude Pacitto et Franck Tordjman}

Volume 13, numéro 3-4, 2000

URI : https://id.erudit.org/iderudit/1008680ar

DOI : https://doi.org/10.7202/1008680ar

Aller au sommaire du numéro

Éditeur(s)

Presses de l'Université du Québec

ISSN

0776-5436 (imprimé)

1918-9699 (numérique)

Découvrir la revue

Citer cet article

Pacitto, J.-C. \& Tordjman, F. (2000). Très petite entreprise et marketing : les causes d'un malentendu. Revue internationale P.M.E., 13(3-4), 37-62.

https://doi.org/10.7202/1008680ar
Résumé de l'article

Souvent ignoré, rarement utilisé, le marketing n'apparaît pas pour les très petites entreprises comme une nécessité. Ce désintérêt s'explique autant par des raisons internes et organisationnelles propres à ces entreprises que par une manière spécifique d'envisager les relations avec l'environnement. C'est précisément à partir des caractéristiques de cette relation que l'on peut saisir le particularisme de la TPE par rapport à la démarche commerciale en général et au marketing en particulier. 


\title{
Très petite entreprise et marketing: les causes d'un malentendu
}

\author{
Jean-Claude PACITTO \\ IUT, Paris XII
}

Franck TORDJMAN

Directeur, Alliance française de Toronto

\section{MOTS CLÉS}

\section{TPE - Marketing - Clientèle - Fidélisation - Commercialisation}

\begin{abstract}
RÉSUMÉ
Souvent ignoré, rarement utilisé, le marketing n'apparaît pas pour les très petites entreprises comme une nécessité. Ce désintérêt s'explique autant par des raisons internes et organisationnelles propres à ces entreprises que par une manière spécifique d'envisager les relations avec l'environnement. C'est précisément à partir des caractéristiques de cette relation que l'on peut saisir le particularisme de la TPE par rapport à la démarche commerciale en général et au marketing en particulier.
\end{abstract}

\section{LES AUTEURS}

Maître de conférences, Jean-Claude Pacitto enseigne la stratégie et l'organisation des entreprises à l'IUT Techniques de commercialisation de l'Université de Paris XII. Ses recherches effectuées dans le cadre des activités du laboratoire IRG management portent sur la gestion des très petites entreprises. Deux domaines sont de ce point de vue privilégiés : le problème de l'innovation dans les très petites structures et celui de la commercialisation. Adresse: 2 , rue de la Fontaine, 89290 Augy, France, téléphone: 01451719 26, télécopieur: 0145171928 , courriel : <pacitto@univ-paris12.fr>

Professeur agrégé d'économie et de gestion, Franck Tordjman a enseigné l'économie et le marketing à l'IUT Techniques de commercialisation de I'Université de Paris XII entre 1993 et 2000 ; il dirige actuellement l'Alliance française de Toronto. Il a été cadre dans des entreprises multinationales de 1983 à 1992 (Procter \& Gamble, Nielsen, Honda). Après avoir connu les très grandes entreprises, ses recherches en cours portent sur la gestion des très petites structures. Adresse: Alliance française, Toronto Downtown, Toronto, Ontario, Canada, M5R 2 S7. 


\begin{abstract}
For tiny companies, marketing though requisite is not a foregone conclusion. While in-house and organizational reasons can provide a partial explanation for this, the special relation between tiny companies and their environment deserve closer attention.
\end{abstract}

Insight can be gained into the tiny approach to business in general and marketing in particular by a deeper understanding of this special relation.

\title{
RESUMEN
}

Frecuentemente ignorado, utilizado raramente, el marketing no parece necessario para las pequenas empresas.

Este desinteres se explica tanto por razones internas y organizacionales propias a esas empresas por una manera especifica de tratar las relaciones con el medio ambiente. Precisamente, a partir de las caracteristicas de esta relacion que podemos tomar el particularismo de la TPE-MPE, en relacion con el ramo comercial en general y el marketing especificamente.

\section{ZUSAMMENFASSUNG}

Oft ignoriert, selten angewendet, erscheint das Marketing für die sehr kleinen Unternehmen (TPE) nicht als Notwendigkeit. Dieses mangelnde Interesse erklärt sich einerseits mit internen und organisatorischen Faktoren, die typisch sind für diese Unternehmensgrösse. Andererseits spielt die Art und Weise eine Rolle, wie die Beziehungen mit der Umwelt betrachtet werden. Diese beiden Gründe bestimmten die Eigenart der sehr kleinen Unternehmen und ihre Marketingphilosophie.

\section{Introduction}

\section{Le marketing concerne-t-il les PME?}

Pensé surtout dans le cadre de la grande entreprise et mettant en œuvre des techniques souvent sophistiquées et donc coûteuses, il devient dès lors légitime de s'interroger sur les possibles applications de la démarche marketing aux PME compte tenu de leurs ressources plus limitées que celles des grandes entreprises et de leurs particularités (Marchesnay, 1988).

De surcrôt, la littérature consacrée au marketing de la PME est peu développée, hétérogène (Herrmann, 1998 ; Perreault, 1994) et il est à noter que les études, dans leur très grande majorité, se concentrent sur les moyennes entreprises, rarement sur les petites entreprises (moins de 50 salariés) et encore plus rarement sur les très petites entreprises (moins de 20 salariés).

De fait et comme Hills (1987) l'avait souligné, le particularisme de ces entreprises devient vite facteur d'exclusion. À ce particularisme structurel s'ajoute, dans certains pays (France, Italie), des spécificités institutionnelles (rattachement de ces 
entreprises au secteur de l'artisanat) qui ne sont pas sans influer sur le comportement des TPE (Auvolat, Lavigne et Mayère, 1985).

Toute réflexion sur le rapport qu'entretiennent les TPE avec le marketing débouche inéluctablement sur la question de leurs relations au marché.

Dans cette perspective, il nous a paru indispensable d'essayer de comprendre, à travers l'étude de la fonction commerciale, quels types de relations les TPE nouent avec leur environnement.

C'est à partir de ce préalable, pensons-nous, qu'on sera le mieux à même de réfléchir sur la nécessité ou non d'un marketing spécifique ou du moins d'outils commerciaux adaptés.

\section{Méthodologie}

\subsection{Considérations générales}

Afin d'appréhender les relations qu'entretiennent les très petites entreprises par rapport à la fonction commerciale et de mesurer autant que possible l'impact de certaines dimensions (comme l'effet dépendance), le choix de la méthode quantitative nous a paru le mieux adapté et, dans cette perspective, ont été privilégiées les entreprises de production directement concernées par la démarche commerciale.

L'échantillon se répartit en trois activités : les imprimeurs (139 entreprises), les prothésistes (dentaires et autres ; 121 entreprises) et les divers (ameublement, habillement, métallurgie ; 116 entreprises). Trois cent vingt-quatre enquêtes ont été effectuées dans la région Île-de-France et 52 en région Franche-Comté.

On considérera l'échantillon comme représentatif tel quel des professions concernées. Compte tenu de la nature des questions posées, il n'était pas nécessaire d'investiguer plus d'entreprises, sauf à vouloir absolument mesurer une éventuelle différence Paris-province; il aurait fallu alors doubler l'échantillon, ce qui n'était pas envisageable. L'intervalle de confiance des résultats sur les proportions modales est de l'ordre de $5 \%$ en plus et en moins, c'est-à-dire qu'une valeur de $40 \%$ peut se lire « il y a $95 \%$ de chances pour que le bon chiffre se situe entre $35 \%$ et $45 \%$ ». L'effectif s'est en tous cas avéré suffisant et les phénomènes en jeu suffisamment typés pour permettre des analyses de variance hautement significatives; la taille moyenne des entreprises est de 14 salariés ; la médiane s'établit à 9 et les deux quartiles à 6 et 17 . Cent quarante-six entreprises ont plus de 10 salariés, soit près de $40 \%$.

Mais seules les tranches de taille sont significatives ; en effet, les chiffres ont été partiellement redressés et proviennent, pour une part, de déclarations directes, pour une autre part, des extractions du répertoire sirène ayant servi à définir la 
population mère de l'échantillon et les deux peuvent diverger. Le répertoire sirène est un instrument administratif obligatoire où doivent être immatriculés toutes les entreprises et établissements soumis à des obligations fiscales et sociales. Les taux de réponse sont d'autant meilleurs que la taille est importante; il faut retenir que l'échantillon est constitué pour les deux tiers des entreprises des métiers, le tiers restant est issu de la population des 11 à 50 salariés et les deux peuvent diverger. Nous avons aussi utilisé dans cette étude certains résultats de l'enquête Time (technology innovation in micro-enterprise) et qui concernait 1016 entreprises artisanales (moins de 10 salariés) de production. En effet, cette enquête visait aussi à explorer l'influence de la clientèle sur les processus innovants, notamment au niveau des impulsions à l'innovation et des structures de clientèle. Les résultats de l'enquête mettent en évidence le rapport privilégié qu'entretiennent les TPE avec leurs clientèles et aussi le fait ( $c f$. section 2) que, pour celles-ci, la clientèle et le marché sont deux réalités souvent confondues.

\subsection{Les opinions des dirigeants des TPE sur la fonction commerciale}

Dans le but de mieux cerner l'attitude des dirigeants des TPE par rapport à la commercialisation, il leur était demandé de se prononcer sur un certain nombre d'opinions. Celles-ci ont émergé après le dépouillement des entretiens exploratoires menés avec des dirigeants de TPE et des responsables d'organisations professionnelles. Dans un premier temps, les énoncés sont examinés successivement, dans l'ordre du mieux accepté au plus souvent rejeté.

Rappelons que les réponses varient sur une échelle de 1 (accord enthousiaste) à 5 (rejet sans appel), le chiffre médian 3 pouvant aussi bien s'interpréter comme de l'hésitation que comme de l'indifférence. De la façon dont les questions sont posées, il y a un biais vers le 1 ; les répondants peu motivés par le sujet ont plus tendance à acquiescer qu'à s'opposer ou même à se placer en position médiane en optant pour une réponse de type neutre ou sans opinion.

Cinq opinions ont ainsi été proposées aux dirigeants des TPE de l'enquête.

« Dans la très petite entreprise, on ne peut pas dissocier l'activité commerciale du reste. Pour établir un devis, l'acte commercial le plus important, il faut tout connaître de la production.»

C'est l'énoncé le plus consensuel (moyenne générale 1,48). On n'observe aucune esquisse de «courbe en $U$ ». Il y a un léger effet négatif sur la taille; plus l'entreprise est grande, plus elle est amenée à prendre quelque distance d'une affirmation qui fait l'unanimité.

«L'avantage de la très petite entreprise, c'est de fournir des services personnalisés face à une demande très diversifiée. C'est pourquoi les outils commerciaux de la 
grande entreprise ne sont pas adaptés, car ils ne sont pas fondés sur une relation personnelle avec le client. »

L'accord est général (moyenne 1,78); on note un petit effet négatif sur la taille et une légère défiance relative du secteur des prothèses. La courbe est globalement en « $L$ »; il s' agit en fait d' un compromis entre le « $U$ », qui apparaît sur les prothèses et la décroissance régulière et rapide qu'on observe chez les imprimeurs.

«Dans notre activité et surtout pour discuter avec le client, le plus important, c'est de bien connaître le métier ; les techniques et les théories qu'on enseigne à l'université, cela vient bien après et on n'en a pas vraiment besoin. »

La tendance générale est favorable, mais la moyenne n'est que de 2,06 , donc légèrement en deçà du seuil correspondant au "plutôt d'accord »; la proposition des « tout à fait d'accord» tombe en dessous des $50 \%$.

La courbe est monotone décroissante chez les imprimeurs, alors que le «U » est marqué dans les deux autres catégories, ce qui le conduit à apparaître nettement sur l'ensemble. L'effet taille est brouillé; seules les très petites entreprises et les imprimeurs se distinguent par un avis plus favorable que la moyenne.

« Pour affronter la concurrence, la très petite entreprise doit grandir et trouver de nouveaux marchés. Pour cela, elle a besoin de techniques commerciales modernes et efficaces. »

Si l'on perçoit un accord d'ensemble, c'est du bout des lèvres (moyenne 2,39 ) ; les réticences les plus fortes se rencontrent chez les plus petites entreprises et chez les prothésistes, et ce, sans doute pour des raisons différentes; il n'y a pas de courbe en « $U$ », mais une décroissance lente, avec un point de départ peu élevé ( $35 \%$ de très favorable) et une forte représentation du centre de la distribution.

«Une très petite entreprise n'a pas les moyens d'avoir un responsable dont ce serait la seule activité; il vaut mieux utiliser une personne extérieure rémunérée à la commission.»

La moyenne est au milieu du spectre $(2,89)$ et on observe une parfaite courbe en « $U$ » bien symétrique; peu d'avis médians et un regroupement sur les deux extrêmes.

\subsection{Classification selon les opinions}

L'algorithme utilisé est celui du minimum de variance de ward, dans la procédure de classification hiérarchique ascendante CLUSTER de SAS.

La normalisation a priori des variables entre 1 et 5 et l'absence de queues de distribution trop fines prévient du risque de points isolés nuisibles à l'interprétation des classes. 
La taille de celles-ci et les possibilités d'interprétation ont primé sur des considérations de seuil de variance expliquée pour fixer leur nombre à six.

Six profils de dirigeants de TPE ont ainsi pu être individualisés :

a) les « défensifs » (36 entreprises): on est ici en présence d'entrepreneurs qui souhaitent conserver le contrôle sur toutes leurs activités (refus du commercial), qui refusent de voir leur entreprise croître et qui restent très attachés aux valeurs de la très petite entreprise (attachement au métier et à la personnalisation des prestations). Pour ces entrepreneurs, la dissociation entre activité commerciale et activité de production n'est pas envisageable ;

b) les « esprit PME » (54 entreprises): au contraire des précédents, les entrepreneurs de ce groupe ne sont pas fermés à la démarche commerciale, mais privilégient les solutions internes (un commercial salarié). Ils souhaitent grandir et rejettent les valeurs de la très petite entreprise, leur modèle restant celui de la moyenne et grande entreprise; la conquête de nouveaux clients et l'utilisation de techniques commerciales modernes sont préférées à une personnalisation des liens avec les clientèles existantes ;

c) les « offensifs» (77 entreprises) : ils partagent beaucoup de choses avec la catégorie précédente (envie de grandir, internalisation de la fonction commerciale), mais s'en détachent sur le problème des valeurs. En effet, ces entrepreneurs restent attachés à celles de la très petite entreprise, notamment pour tout ce qui touche à la relation à la clientèle et au lien qui est fait entre services personnalisés et avantage concurrentiel de la très petite entreprise ;

d) les «modernes » (45 entreprises): ces entrepreneurs dissocient l'acte de production de celui de commercialisation. Ils sont à la recherche de nouvelles clientèles et sont par là favorables aux techniques qui leur permettront de les capter;

$e)$ les «protégés » (81 entreprises) : ils sont assez proches des entrepreneurs défensifs, mais se montrent moins réticents à la démarche commerciale (internalisation); toutefois, ils ne dissocient pas (comme les défensifs) l'acte de production de l'acte commercial. Ils n'ont pas d'opinion tranchée quant à l'utilisation éventuelle de personnes extérieures rémunérées à la commission ;

f) les «passifs » (78 entreprises) : ces entrepreneurs n'ont pas d'opinion marquée, mais restent attachés aux valeurs de la TPE (attachement au métier et à la personnalisation des services). 


\section{Les obstacles à l'adoption d'une démarche marketing}

\subsection{La confusion entre marché et client}

La réserve globale des TPE face à une démarche marketing trouve son origine dans la perception même qu'ont les dirigeants du marché et leur réticence à mobiliser des ressources en vue de conquérir de nouveaux clients. Marchesnay (1988) et Polge (1996) ont montré que la notion de concurrence n'était pas familière aux petites entreprises et que, même au niveau du vocabulaire employé, ce terme apparaissait peu au contraire de ceux de collègue ou de partenaire. D'un point de vue institutionnel, il est évident que le rattachement des TPE au secteur de l'artisanat (qui regroupe en France les entreprises de moins de 10 salariés) n'a pas été sans conséquences sur le développement de cultures d'entreprises " particularistes ». Le secteur de l'artisanat s'est, en effet, structuré autour du rejet d'une certaine forme d'économie de marché et d'une méfiance à l'égard des phénomènes de concurrence et d'adaptation (David, 1998). Par là, l'artisanat institutionnel a préféré mettre l'accent sur la spécificité des valeurs plutôt que sur les seuls arguments économiques (l'effet taille par exemple). Cela n'a d'ailleurs pas été sans influer sur le type d'études consacré aux entreprises de ce secteur. Ainsi, si l'on trouve nombre de monographies mettant en relief la spécificité culturelle de l'artisanat, on ne rencontre, par contre, que peu d'études, qu'elles soient de nature qualitative ou quantitative, qui traitent des problèmes économiques ou de gestion. On peut penser que la crainte d'une normalisation avec les autres entreprises et la fin, par conséquent, des spécificités structurelles de l'artisanat ont largement favorisé cet état de fait et contribué à façonner un état d'esprit où les préoccupations économiques n'ont pas toujours dominé. De ce point de vue, les stratégies de créneau et de niche sont souvent perçues, de la part de ces entreprises, comme un moyen d'éviter cette confrontation qui, à leur avis, ne les concerne pas.

L'insertion dans l'environnement ne résulte pas, pour elles, d'une évaluation des offres de la part du demandeur, mais de la rencontre d'un savoir-faire unique et d'une demande explicite. C'est pourquoi la notion de marché apparaît comme étrangère à leur culture. Pourtant, il nous a tout de même paru intéressant de garder dans le contenu de notre enquête un questionnement ayant trait au marché et sans chercher à adapter ce terme aux réalités de la TPE. Il nous est apparu que c'était là la meilleure façon de percevoir ce que précisément ces entreprises entendaient par «marché ».

La quasi-totalité des entreprises de notre échantillon déclarent connaître leur marché, $80 \%$ d'entre elles estimant ne pas avoir besoin d'études de marché (tableau 1). Ce chiffre ne doit pas faire illusion; il nous laisse penser que sont, de fait, confondues deux réalités : le client et le marché. En effet, le résultat semble 
TABLEAU 1

Connaissance du marché

\begin{tabular}{|c|c|c|c|c|c|c|c|c|c|}
\hline & \multicolumn{4}{|c|}{ Marché bien connu ? } & \multicolumn{5}{|c|}{ Besoin d'une étude de marché ? } \\
\hline & & Non & & Oui & & Non & & Oui & Total \\
\hline & $\mathbf{N}$ & $\%$ & $\mathbf{N}$ & $\%$ & $\mathbf{N}$ & $\%$ & $\mathbf{N}$ & $\%$ & $\mathbf{N}$ \\
\hline Total & 25 & 6,6 & 351 & 93,4 & 304 & 80,9 & 72 & 19,1 & 376 \\
\hline $\begin{array}{l}\text { Activité } \\
\text { Divers } \\
\text { Imprimeurs } \\
\text { Prothèses }\end{array}$ & $\begin{array}{r}5 \\
13 \\
7\end{array}$ & $\begin{array}{l}4,3 \\
9,4 \\
5,8\end{array}$ & $\begin{array}{l}111 \\
126 \\
114\end{array}$ & $\begin{array}{l}95,7 \\
90,6 \\
94,2\end{array}$ & $\begin{array}{r}90 \\
117 \\
97\end{array}$ & $\begin{array}{l}77,6 \\
84,2 \\
80,2\end{array}$ & $\begin{array}{l}26 \\
22 \\
24\end{array}$ & $\begin{array}{l}22,4 \\
15,8 \\
19,8\end{array}$ & $\begin{array}{l}116 \\
139 \\
121\end{array}$ \\
\hline $\begin{array}{l}\text { Taille } \\
1 \text { à } 6 \\
7 \text { à } 10 \\
11 \text { à } 20 \\
\text { Plus de } 20\end{array}$ & $\begin{array}{r}9 \\
11 \\
3 \\
2\end{array}$ & $\begin{array}{l}9,3 \\
8,3 \\
3,8 \\
3,0\end{array}$ & $\begin{array}{r}88 \\
122 \\
77 \\
64\end{array}$ & $\begin{array}{l}90,7 \\
91,7 \\
96,3 \\
97,0\end{array}$ & $\begin{array}{r}84 \\
102 \\
67 \\
51\end{array}$ & $\begin{array}{l}86,6 \\
76,7 \\
83,8 \\
77,3\end{array}$ & $\begin{array}{l}13 \\
31 \\
13 \\
15\end{array}$ & $\begin{array}{l}13,4 \\
23,3 \\
16,3 \\
22,7\end{array}$ & $\begin{array}{r}97 \\
133 \\
80 \\
66\end{array}$ \\
\hline $\begin{array}{l}\text { Opinions } \\
\text { Passifs } \\
\text { Offensifs } \\
\text { Défensifs } \\
\text { Esprit PME } \\
\text { Modernes } \\
\text { Protégés }\end{array}$ & $\begin{array}{l}6 \\
1 \\
4 \\
3 \\
4 \\
7\end{array}$ & $\begin{array}{r}7,6, \\
1,3 \\
11,1 \\
5,4 \\
8,9 \\
8,5\end{array}$ & $\begin{array}{l}73 \\
77 \\
32 \\
53 \\
41 \\
75\end{array}$ & $\begin{array}{l}92,4 \\
98,7 \\
88,9 \\
94,6 \\
91,1 \\
91,5\end{array}$ & $\begin{array}{l}64 \\
56 \\
32 \\
44 \\
43 \\
65\end{array}$ & $\begin{array}{l}81 \\
71,8 \\
88,9 \\
78,6 \\
95,6 \\
79,3\end{array}$ & $\begin{array}{r}15 \\
22 \\
4 \\
12 \\
2 \\
17\end{array}$ & $\begin{array}{r}19 \\
28,2 \\
11,1 \\
21,4 \\
4,4 \\
20,7\end{array}$ & $\begin{array}{l}79 \\
78 \\
36 \\
56 \\
45 \\
82\end{array}$ \\
\hline
\end{tabular}

Source : Enquête IRG.

démontrer que le concept de marché a pour les TPE une toute autre signification que pour les moyennes et grandes entreprises et qu'il n'est pas synonyme d'espace à conquérir. En répondant massivement et positivement à cette question, elles montrent qu'au fond celle-ci n'a pas de sens pour elles. La seule réalité qui s'impose à elles est celle du client et, comme on le verra, du client déjà conquis.

La réponse négative formulée à l'égard des études de marché laisse entrevoir que la conquête de nouvelles cibles ne les intéresse tout simplement pas. Ainsi, l'énoncé d'opinion concernant la nécessité de trouver de nouveaux clients pour affronter la concurrence est-il le moins consensuel, les plus petites entreprises étant les plus réticentes, rejetant même cette proposition ( $c f$. section 1.2.). De la même façon, l'étude de la concurrence n'est pas jugée utile, celle-ci étant souvent perçue comme située dans un « ailleurs » difficilement localisable. On remarquera aussi que le profil de l'entrepreneur ne modifie pas en profondeur la réponse apportée à la nécessité ou non d'études de marché. Dans cette perspective, il nous avait paru utile, lors d'une précédente étude sur l'innovation technologique dans les TPE, d'isoler les exigences propres de la clientèle et de nous démarquer de l'opposition traditionnelle et théorique entre «demand pull» et «technology push », ce qui nous 
eut exposés à des résultats déconcertants. On ne peut, en effet, douter que celle-ci repose sur une conception du marché qui est celle de la moyenne et grande entreprise. La clientèle n'étant pas considérée par la TPE comme une force de marché parce qu'étant le plus souvent déjà conquise. De fait, ce sont les exigences de la clientèle qui constituent, pour la TPE, la première impulsion à l'innovation, les pressions de la concurrence n'obtiennent, elles, que la troisième et dernière position (Médus et Pacitto, 1994). C'est cette clientèle qu'il faut, dès lors, connaître. Cette investigation doit nous conduire à l'identification la plus précise possible des clientèles des TPE. Les résultats de l'enquête innovation menée sur les TPE industrielles apportent sur ce point des éclairages importants. On remarque ainsi que les TPE ont d'autant plus de chances d'innover qu'elles sont en contact avec des clientèles technologiquement exigeantes (celles par exemple liées au secteur de la défense ) et de taille plus élevée (PME ou autres). Ce qui démontre que le processus innovant dans la TPE est fréquemment déclenché par le client, qu'il n'est pas de nature proactive (Kaminski, 1993) et qu'il vise rarement à repositionner l'entreprise.

La non-orientation «marché » des TPE tient pour beaucoup à leur mode d'insertion dans l'environnement et à la nature du processus de création des ressources. Ce dernier est, pour la TPE, étroitement dépendant des demandes qui lui sont adressées. La TPE raisonne en fonction de cette demande et non pas en fonction d'un éventuel positionnement du produit par rapport à des demandes plus indifférenciées ou par rapport à des offres concurrentes. De toute façon, ce processus de création de ressources est tellement orienté et, par là même dépendant, que son adaptation à d'autres demandes nécessiterait un effort d'investissement que très peu de TPE seraient susceptibles d'assumer. Enfin, on ne doit pas sous-estimer l'un des problèmes fondamentaux des dirigeants de TPE : la gestion du temps lié à la pluralité des rôles qu'ils assument (Candau, 1981). Tout cela explique, en dernier lieu, l'origine des phénomènes de dépendance à la clientèle ( $c f$. section 2.). Ces entreprises préfèrent investir lorsque le débouché est assuré, la demande explicite. Ces constatations nous amènent à douter de la nécessité «d'immerger» les TPE dans des espaces concurrentiels « ouverts ». Loin d'être toujours une source de compétitivité, cette immersion serait plus sûrement génératrice de résultats catastrophiques ; elle conduirait à un éloignement des TPE de leurs clientèles habituelles qui constituent non seulement des débouchés assurés, mais aussi des sources de compétitivité fondamentales. C'est cette proximité qui permet aux TPE d'assurer une certaine cohésion stratégique (Porter, 1997), cohésion qui assure la pérennité.

Une autre difficulté surgit lorsqu'on examine le rapport des TPE au marché : celle du savoir-faire (Siméoni, 1998), celui-ci étant plus envisagé comme un tour de main, une référence idenditaire (Letowski, 1987) que comme une compétence fondamentale, un cœur de métier (Deret, 1999) exploitable sur d'autres applications. Souvent ce savoir-faire se confond avec le produit, ce qui rend encore plus difficile la dissociation des deux. 
Ce qui fait le plus défaut à la TPE ce n'est pas, en dernier lieu, ce fond de compétence centrale (Amendola et Gaffard, 1988), mais la conscience d'en posséder un et la conscience qu'il pourrait déboucher sur d'autres applications, ne serait-ce que dans le cadre de la relation privilégiée avec la clientèle. Toute démarche commerciale devra nécessairement passer par ce préalable, définir ce que l'on peut faire et à partir de là examiner les éventuels nouveaux débouchés. Il s'agit au fond de convaincre l'entrepreneur que son savoir-faire, d'une part, ne se confond pas avec le produit, qu'il constitue une compétence qui peut être déployée ailleurs et, d'autre part, que le produit doit pouvoir faire l'objet, de la part du client, d'une évaluation. La notion de satisfaction doit être pensée réciproquement et non plus seulement comme la rencontre unilatérale entre un savoir-faire et un besoin donné. Il faudra s'appliquer à définir au mieux les compétences à l'œuvre et envisager, à partir de là, les types d'application qui pourraient en résulter et, de cette manière, les clientèles qui pourraient être servies. C'est seulement lorsque ce préalable sera effectué que l'on pourra songer à examiner les demandes spécifiques du marché. De ce point de vue, on peut penser que la communication commerciale de la TPE devra plus porter sur la ou les compétences centrales de l'entreprise, d'autant plus que, bien souvent, les TPE sont en position de sous-traitance et qu'elles n'ont pas, ou peu, de contacts avec le consommateur final.

\subsection{La vente aux mains du dirigeant}

Qui, dans la TPE, a concrètement en charge la commercialisation des produits (tableau 2) ? Deux types d'interrogation surgissent alors à la lecture des résultats : concernant le faible taux de commerciaux nettement individualisés, est-il la conséquence de la faible différenciation de ces structures, qui conduit le chef d'entreprise à assumer plusieurs fonctions ou résulte-t-il d'une attitude générale à l'égard de la fonction commerciale? Nous examinerons ce point dans un second temps.

Si le recours à un commercial est clairement corrélée avec la taille de l'entreprise (le seuil se situant autour de 10 salariés ; au-delà, on note un début de différenciation structurelle), il n'en demeure pas moins que, dans plus de $80 \%$ des entreprises de moins de 6 personnes, soit c'est l'entrepreneur seul qui se charge de la commercialisation, soit la fonction n'est pas identifiée et il n'y a donc pas de collaborateurs spécialisés (tableau 2).

Sur l'ensemble des entreprises de moins de 10 personnes, on ne peut qu'être frappé par le fait que les deux tiers des entrepreneurs assument seuls la fonction commerciale. Cela nous renvoie à l'opinion générale très répandue dans ces firmes selon laquelle, pour commercialiser les biens, il faut d'abord connaître la production, le métier. Cet item d'opinion (opinion 5) est, en effet, le plus consensuel de notre enquête, seules les plus grandes entreprises prenant quelques distances avec cette affirmation. 
TABLEAU 2

Qui s'occupe de la fonction commerciale?

\begin{tabular}{lrrrrrrrrrr}
\hline & $\begin{array}{c}\text { Fonction } \\
\text { non identifiée }\end{array}$ & $\begin{array}{c}\text { Le patron } \\
\text { tout seul }\end{array}$ & $\begin{array}{c}\text { Quelqu'un } \\
\text { en interne }\end{array}$ & $\begin{array}{c}\text { Un } \\
\text { prestataire } \\
\text { externe }\end{array}$ & Total \\
\hline & $\mathbf{N}$ & $\%$ & $\mathbf{N}$ & $\%$ & $\mathbf{N}$ & $\mathbf{\%}$ & $\mathbf{N}$ & $\%$ & $\mathbf{N}$ \\
\hline Total & $\mathbf{7}$ & 19,7 & 163 & 43,4 & 117 & 31,1 & 22 & 5,9 & 376 \\
\hline Activité & & & & & & & & & \\
Divers & 21 & 18,1 & 49 & 42,2 & 41 & 35,3 & 5 & 4,3 & 116 \\
Imprimeurs & 17 & 12,2 & 54 & 38,8 & 57 & 41,0 & 11 & 7,9 & 139 \\
Prothèses & 36 & 29,8 & 60 & 49,6 & 19 & 15,7 & 6 & 5,0 & 121 \\
\hline Taille & & & & & & & & & \\
1 à 6 & 24 & 24,7 & 55 & 56,7 & 14 & 14,4 & 4 & 4,1 & 97 \\
7 à 10 & 30 & 22,6 & 63 & 47,4 & 34 & 25,6 & 6 & 4,5 & 133 \\
11 à 20 & 15 & 18,8 & 27 & 33,8 & 36 & 45,0 & 2 & 2,5 & 80 \\
Plus de 20 & 5 & 7,6 & 18 & 27,3 & 33 & 50,0 & 10 & 15,2 & 66 \\
\hline Opinions & & & & & & & & & \\
Passifs & 15 & 19 & 39 & 49,4 & 22 & 27,8 & 3 & 3,8 & 79 \\
Offensifs & 9 & 11,5 & 29 & 37,2 & 35 & 44,9 & 5 & 6,4 & 78 \\
Défensifs & 6 & 16,7 & 19 & 52,8 & 10 & 27,8 & 1 & 2,8 & 36 \\
Esprit PME & 8 & 14,3 & 15 & 26,8 & 26 & 46,4 & 7 & 12,5 & 56 \\
Modernes & 12 & 26,7 & 19 & 42,2 & 13 & 28,9 & 1 & 2,2 & 45 \\
Protégés & 24 & 29,3 & 42 & 51,2 & 11 & 13,4 & 5 & 6,1 & 82 \\
\hline
\end{tabular}

Source : Enquête IRG.

Pour la majorité de ces entrepreneurs, la prise en charge de la commercialisation des produits par une personne spécifique a un inconvénient majeur: elle atténue le caractère technique de ces derniers et sous-valorise le savoir-faire à l'origine du produit. De la même façon, le recours à un commercial atténuerait la relation qu'entretient le dirigeant de la TPE avec ses clients, relation dont la personnalisation est perçue comme l'avantage même de la TPE, sa caractéristique distinctive majeure (Auvolat, Lavigne et Mayere, 1985). Cette relation privilégiée que le dirigeant souhaite maintenir s'explique aussi par le fait qu'il s'estime seul compétent pour retranscrire en actes opérationnels les modifications ou les nouvelles exigences demandées par le client. Dans la négociation, le dirigeant perçoit le comment ou les possibilités d'effectuer ces modifications et il pense que les remontées des commerciaux ne pourraient relier aussi facilement les exigences des clients et l'évolution rendue nécessaire du savoir-faire. Au-delà des spécificités des dirigeants des TPE, il y a là un véritable problème qui se trouve posé et qui explique aussi, en partie, la grande réticence de ces petites structures à embaucher des jeunes diplômés. Les dirigeants des TPE éprouvent beaucoup de difficultés à faire évoluer leurs compétences et, plus globalement, leur culture d'entreprise. Les 
compétences, souvent façonnées par la relation privilégiée avec un ou quelques clients, se figent rapidement et créent une culture d'entreprise qu'il est difficile de changer. Dans le système de valeurs des TPE, si la disponibilité au client apparaît comme primordiale, on ne trouve néanmoins pas de motivations liées à une volonté d'engagement dans des champs concurrentiels (Pacitto, 1996).

Une enquête menée par la Sofrès (1995) a pu ainsi mettre en évidence que le besoin d'indépendance restait, pour les entreprises de moins de 20 salariés, la motivation principale à l'origine de la création de l'entreprise. La réticence souvent constatée des petites entreprises à l'égard de tout ce qui touche à la fonction commerciale peut s'expliquer par la difficulté qu'elles éprouvent à se situer par rapport à la concurrence. L'idée même de positionnement apparaît comme étrangère à la majorité de ces entreprises. La perception du positionnement est indissociable de celle de l'univers concurrentiel dans lequel on se situe. Or, précisément et comme nous avons tenté de le montrer, les dirigeants des petites entreprises tendent à évacuer celui-ci. La recherche de la pérennité passe plus sûrement par un renforcement des liens avec la clientèle existante que par l'exploration de nouveaux débouchés ou la prise en compte des offres concurrentes.

Il est aussi intéressant de constater comment le lien intime qui se crée entre l'entrepreneur et son savoir-faire conduit à une évaluation toute subjective des produits fabriqués, de leur unicité supposée et plus généralement des savoir-faire à l'œuvre dans l'entreprise. On remarque ainsi que sont souvent confondues deux choses : la différenciation dans la manière de concevoir ou fabriquer un produit et le produit en lui-même. Si la première distingue fréquemment les TPE des autres entreprises et, notamment celles de plus grande dimension, il est moins sûr que les produits soient, eux aussi, toujours différenciés. De surcroît, les dirigeants des TPE ne souhaitent pas voir leur entreprise croître, croissance qui est associée à une perte de contrôle sur l'entreprise. Cette croissance ne doit pas être comprise uniquement en des termes quantitatifs. Ainsi, pour ces entrepreneurs, le début de différenciation structurelle apparaît aussi comme un phénomène de normalisation et donc de perte de contrôle, surtout lorsque cela touche au devenir du produit. La délégation s'accorde mal avec ces valeurs d'accomplissement de même qu'avec celle de bel ouvrage. Enfin, cette difficulté à déléguer résulte pour une bonne part du mode de constitution des compétences des TPE. Peu formalisées, acquises progressivement, elles ne se distinguent pas, ou très peu, de l'exercice de l'activité de l'entrepreneur. En ce sens, toute perspective d'agrandissement est ressentie comme une perte d'autonomie et d'identité.

Il est évident que l'embauche d'un commercial nécessiterait un effort de formalisation que le petit entrepreneur ne peut ou ne souhaite pas assumer. Ainsi, le caractère tacite des savoirs (Polanyi, 1966; Reix, 1995) «we can do more than we can tell» apparaît comme un frein à l'adjonction de nouvelles compétences. 
Organisation «mémorisante» (Girod, 1995), la petite entreprise « apprend » en capitalisant des savoirs d'origine diverse, capitalisation qui s'effectue bien souvent, à travers le canal du seul dirigeant d'entreprise.

De plus, comme le montre Pitcher (1996), la transmission du savoir artisanal se fonde sur la pratique, l'expérience et la «connaissance tacite » et elle s'opère dans un contexte de soumission à l' autorité qui paraît antinomique avec la délégation que nécessite le recours à un commercial. Mais le caractère tacite des savoirfaire a une autre conséquence, soit de rendre plus difficile une éventuelle communication commerciale. En effet, la difficulté à formaliser ces savoir-faire constitue aussi un frein qui ne doit pas être négligé lorsqu'on préconise des politiques commerciales en faveur de ces entreprises. Toute démarche commerciale suppose une description la plus précise possible de ce que l'on sait faire, de ce que l'on fabrique ou de ce que l'on peut fabriquer. Or, cette description s'avère, dans le cas des petites structures, problématique. C'est pour cette raison que les dirigeants préfèrent le contact personnel à une communication formelle. Dans cette approche, il y a toujours l'idée de faire partager un savoir-faire, plus que d'en montrer objectivement les avantages. On comprend mieux, dès lors, pourquoi ces dirigeants sont aussi réticents à dissocier la fabrication du produit de sa commercialisation. Cette dissociation les obligerait, en effet, à mettre en avant des qualités du produit mais cette fois-ci évaluées par rapport à ceux de la concurrence, ce qui pour plusieurs semble impossible parce qu'ils ne les connaissent tout simplement pas.

\subsection{Satisfaire plutôt que conquérir}

Le raisonnement en termes de marché apparaît donc, pour la TPE, comme problématique. Sa relation à l'environnement, la TPE ne l'envisage pas autour de la notion d'adaptation, nous entendons par là adaptation à des demandes plus ou moins indifférenciées. Comme le souligne Polge (1996), le marketing est pensé simplement comme une aide visant à faciliter l'insertion d'une capacité dans l'environnement. Dans ce cas, continue le même auteur, il s'apparente plus à une démarche commerciale. Ce qu'il faut bien saisir, c'est que cette réflexion est menée par rapport à une capacité déjà existante. Il n'y a pas et ne peut y avoir réflexion afin de faire évoluer cette capacité et de l'adapter aux divers besoins. Dans cette perspective, le besoin d'investigation de nouveaux clients, de nouvelles cibles n'est que rarement ressenti. Le dirigeant de la TPE raisonne autour d'un savoir-faire déjà constitué et pas, a priori, autour de capacités qu'il lui faudrait posséder pour asseoir un avantage concurrentiel quelconque. Comme nous avons essayé de le montrer, les espaces dans lesquels se meuvent les TPE sont structurés autour de réalités concrètes, identifiables et avec lesquelles elle est le plus souvent en relation.

De ce point de vue, le raisonnement en termes de menaces et d'occasions d'affaires n'apparaît pas adapté. Ces menaces et occasions d'affaires s'évaluent 
toujours par rapport à des clients avec lesquels on est déjà en contact, pas sur la base de scénarios hypothétiques. Le processus d'ajustement, par rapport à la demande, se structure autour de clients et de spécifications explicites.

Dans cette perspective, la démarche commerciale de la TPE ne consistera pas à élargir une offre en proposant une prestation sur des bases strictement concurrentielles, mais le plus souvent à répondre aux exigences du client envisagé comme débouché fondamental.

La notion d'apprentissage par le marché (Dickson, 1992 ; Day, 1994 ; Sinkula, 1994) nous paraît, du point de vue de ces entreprises, comme ambiguë, et certains auteurs (Minguzzi et Passaro, 1995) assimilent trop facilement exigences et caractéristiques des clients avec la sélectivité des marchés. L'approche «écologie des populations » qui sous-tend nombre de ces études finit par occulter l'essentiel en ne cherchant pas à comprendre, en dernier lieu, ce qui fait que ces entreprises ne se préoccupent pas du marché. Lorsque ce problème est abordé, il est à noter qu'il l'est souvent par le biais des profils d'entrepreneurs, ce qui a pour conséquence de créer une césure toute artificielle entre entreprises qui seraient «dans le coup » et celles qui ne le seraient pas, causalité réductrice et en dernière instance peu explicative. Considérer les entreprises peu concernées par les phénomènes de concurrence comme des anomalies ou des entreprises, à terme, condamnées ne résout pas le problème. De plus, on ne doit pas confondre les spécificités des secteurs dans lesquels les entreprises évoluent et les pressions de la concurrence. À cet égard, la notion de paradigme technologique (Dosi, 1984) parait plus pertinente, car moins réductrice. L'objectif des dirigeants des TPE est de préserver la régularité en maximisant la satisfaction plutôt que d'élargir l'offre. La satisfaction passe par un service personnalisé que les TPE opposent volontiers à l'offre standard des grandes entreprises. Ce service personnalisé est associé à la qualité de la production proposée, qualité que l'on juge distinctive de l'offre indifférenciée de la grande entreprise.

Les TPE, en effet, souscrivent largement à l'opinion selon laquelle l'avantage de la TPE réside dans l'offre de services personnalisés et que, par conséquent, les outils commerciaux de la grande entreprise ne sont pas adaptés, car non fondés sur cette relation personnelle avec le client (opinion 2, $c f$. section 1.2.). La personnalisation, c'est aussi pour les TPE la capacité de trouver de nouvelles solutions pour satisfaire aux nouvelles exigences du client, de faire évoluer une prestation en testant un nouveau savoir-faire ou de nouvelles applications. De ce point de vue, les nouveaux besoins du client sont souvent considérés comme des éléments positifs, des incitations à réfléchir à de nouvelles façons de faire. À ce moment précis de sa relation avec le client, le dirigeant de la TPE essaie de fidéliser en accroissant sa capacité d'accompagnement, ce qui est, somme toute, assez éloigné de la démarche marketing standard. 
Il n'y a pas pour autant rejet en bloc de celui-ci ; ainsi l'item d'opinion selon lequel les théories et pratiques commerciales ne sont guère utiles ne bénéficie pas d'un assentiment général. Les dirigeants des TPE, dans leur majorité, n'éprouvent pas d'hostilité à l'égard du marketing, ils estiment qu'il ne les concerne pas. Une plus grande sensibilité au problème ne changerait, pensons-nous, que peu de chose à cet état de fait. Ce qui importe pour ces entreprises, c'est de créer une relation durable avec le client, être à son écoute et on le sera d'autant plus que l'on s'estime être en pleine possession de son métier. Si cette maîtrise s'atténue, la perte de la clientèle apparaît comme inévitable. De surcroît, si l'on projette, par le questionnement, le dirigeant de la TPE dans l'univers concurrentiel, il acquiesce volontiers sur la nécessité de techniques commerciales. Ce qui prouve, en dernier lieu, que l'attitude par rapport au commercial est aussi directement liée au contexte et que celle des TPE n'est que rarement structurée par des phénomènes de concurrence. C'est ce qui explique, comme on le verra, que les outils de captation de nouvelles clientèles (publipostages, fichiers de prospection) soient peu usités. De la même façon, on ne sera pas surpris que $80 \%$ des entreprises de l'échantillon estiment maîtriser les techniques commerciales que nécessite leur activité et qu'elles ne désirent pas s'adjoindre un «jeune commercial».

La recherche de la régularité ne trouve pas seulement son origine dans la poursuite de stratégies de sécurisation; elle apparaît plutôt comme une nécessité économique qui va permettre une utilisation maximale des ressources qui ne pourraient que difficilement être engagées dans des environnements dominés par l'incertitude. Comme pour le cas de l'innovation technologique, l'engagement des ressources dépend du degré de stabilité. La volatilité et l'incertitude qu'elle engendre ne sont pas toujours, dans le cas de la TPE, des déterminants de la compétitivité. L'adoption d'une démarche commerciale obligerait la TPE à renoncer à cette stabilité et déboucherait sur une dispersion des ressources qui pourrait devenir préjudiciable. De surcroît, elle obligerait beaucoup de TPE à se développer, donc à grandir, ce que la majorité d'entre elles se refusent à envisager.

Si l'on veut réfléchir à un futur mercatique possible pour la TPE, il est nécessaire, pensons-nous, d'essayer de relativiser la prédominance technique de l'échange entre celle-ci et son client; de ce point de vue, l'adoption d'une démarche qualité axée sur la satisfaction du client aurait des incidences positives. En effet, comme le remarque Deret (1999), la qualité de la relation avec le client passe par la « chaîne qualité » : accueil, écoute, réactivité, contenu technique, aspect relationnel, devis (formalisation, négociation, finalisation), fichier client, suivi de trésorerie. Ce passage a toujours des conséquences ( $c f$. section 3.3.) sur les compétences de l'entrepreneur, sur son cœur de métier et l'oblige souvent à élargir la conception qu'il se fait de ce dernier, envisagé la plupart du temps autour de la seule compétence technique, du seul savoir-faire. 


\section{La dépendance à la clientèle : une caractéristique majeure de la TPE}

Si le marché se confond bien souvent pour la TPE aux clients déjà conquis, il importe de bien comprendre comment concrètement elle gère sa relation à la clientèle. Dans cette perspective, nous tenterons de «mesurer» l'effet dépendance (Marchesnay, 1994) si caractéristique des petites entreprises. C'est à travers l'indice de concentration de la clientèle que nous le ferons, de même que, dans un second temps, nous essaierons d'évaluer le degré de régularité de celle-ci.

\subsection{La réalité du phénomène}

TABleaU 3

Concentration de la clientèle : valeurs moyennes Moyennes pondérées par l'effectif salarié

\begin{tabular}{cccc}
\hline \multicolumn{4}{c}{ CHIFFRE D'AFFAIRES DES TROIS PLUS GROS CLIENTS } \\
\hline DIVERS & IMPRIMEURS & PROTHÈSES & MOYENNE \\
\hline $43,62 \%$ & $38,81 \%$ & $35,33 \%$ & $39,25 \%$ \\
\hline
\end{tabular}

Source : Enquête IRG.

Quarante pour cent du chiffre d'affaires des TPE est généré par les trois plus gros clients ; ce chiffre montre une mobilisation des ressources assez orientée, voire focalisée. De fait, le déséquilibre des clientèles est évident, ce qui, a contrario, prouverait le caractère résiduel et non stratégique d'une grande partie des clients déclarés. C'est pour les TPE des secteurs traditionnels que cette concentration est la plus marquée, ce qui est somme toute logique puisque c'est là que s'y développe le plus la sous-traitance de capacité.

\section{TABLEAU 4}

Concentration de la clientèle : valeurs moyennes Moyennes pondérées par l'effectif salarié

\begin{tabular}{cccc}
\hline \multicolumn{4}{c}{ CHIFFRE D'AFFAIRES DES TROIS PLUS GROSSES COMMANDES } \\
\hline DIVERS & IMPRIMEURS & PROTHÈSES & MOYENNE \\
\hline $36,37 \%$ & $21,72 \%$ & $31,38 \%$ & $29,82 \%$ \\
\hline
\end{tabular}

Source : Enquête IRG.

Si l'on raisonne cette fois au niveau des commandes, la même constatation s'impose. La part générée par les trois plus grosses d'entre elles représente près de $30 \%$ du chiffre d'affaires. On notera le résultat des imprimeurs (23\%) qui 
prouverait une plus grande fluidité de la clientèle et, corrélativement, un plus grand équilibrage. Ce qui traduit aussi certainement, pour les entreprises de ce secteur, des possibilités beaucoup plus réduites de sous-traitance capacitaire, celles-ci n'étant, pour les raisons déjà évoquées, que rarement des structures de faisabilité pour les plus grandes. Il n'y a pas coopération, ni stricte dépendance, parce que les entreprises, quelle que soit leur taille, s'affrontent avec des ressources quasi identiques (ce qui ne signifie pas équilibrées) dans la captation d'un client, là aussi, identique (l'entreprise quelle que soit sa taille). Cette particularité ne saurait toutefois être interprétée dans le sens d'une plus grande volatilité de la clientèle des imprimeurs, la plus grande dispersion étant régulée par des choix très orientés dans la distribution des ressources.

TABLEAU 5

Concentration de la clientèle : valeurs moyennes Moyennes pondérées par l'effectif salarié

\begin{tabular}{cccc}
\hline \multicolumn{4}{c}{ PROPORTION DE CLIENTS RÉGULIERS } \\
\hline DIVERS & IMPRIMEURS & PROTHÈSES & MOYENNE \\
\hline 58,61 & 65,61 & 65,81 & 63,34 \\
\hline
\end{tabular}

Source : Enquête IRG.

TABLEAU 6

Concentration de la clientèle : valeurs moyennes Moyennes pondérées par l'effectif salarié

\begin{tabular}{cccc}
\hline \multicolumn{4}{c}{ PROPORTION EN CHIFFRE D'AFFAIRES DES CLIENTS RÉGULIERS } \\
\hline DIVERS & IMPRIMEURS & PROTHÈSES & MOYENNE \\
\hline 60,36 & 78,31 & 79,91 & 72,86 \\
\hline
\end{tabular}

Source : Enquête IRG.

La régularité, dans la relation à la clientèle, constitue une caractéristique majeure de la TPE (tableau 5); régularité qui se concrétise dans la réalisation du chiffre d'affaires (tableau 6), puisque les clients réguliers représentent $70 \%$ du chiffre d'affaires de ces entreprises. Cela démontre pour la TPE une certaine stabilité dans sa relation à l'environnement. De la sorte, plutôt que d'une réactivité souvent affirmée (Piore et Sabel, 1984), rarement prouvée, c'est plus certainement dans l'accompagnement stratégique des clientèles que la TPE va chercher sa pérennité. L'accompagnement des clientèles diffère fondamentalement des stratégies classiques d'adaptation.

En effet, les phénomènes d'adaptation doivent être évalués par rapport à la capacité des entreprises à s'adapter à des environnements connus ou moins connus, 
ce qui n'est pas le cas de la TPE. De la même façon, la réactivité doit s'évaluer sur la capacité de l'entreprise à suivre les tendances générales d'un secteur ou d'un marché particulier, ce qui n'est pas le cas des TPE. C'est donc de sa capacité à gérer cet accompagnement que dépendra en dernier lieu sa survie; si réactivité il y a, elle s'opère par rapport à des clients particuliers et non par rapport à un marché.

Le processus d'adaptation se met en branle lorsqu'une exigence particulière est émise, c'est à ce moment que le dirigeant amorcera un processus de réflexion et qu'il réfléchira à de nouvelles applications.

On semble oublier que la forte capacité d'accompagnement des petites entreprises se situant dans les « fameux » districts résulte, pour beaucoup, de cette proximité et de l'intime connaissance qui lient entreprises «pivot » et entreprises membres du réseau. Il n'est donc pas surprenant de constater que, dans le système de valeurs des dirigeants des très petites entreprises, c'est la capacité à répondre rapidement qui obtient la meilleure moyenne, loin devant les items d'opinion exprimant plus les références culturelles classiques de l'artisanat (indépendance, plaisir de la belle ouvrage, etc.).

Tableau 7

Actions commerciales en entreprise

\begin{tabular}{|c|c|c|c|c|c|c|c|c|c|}
\hline & \multicolumn{4}{|c|}{ Plaquette? } & \multicolumn{4}{|c|}{ Publipostage? } & \multirow{3}{*}{$\begin{array}{c}\text { Total } \\
\mathbf{N}\end{array}$} \\
\hline & & Von & & Oui & & Von & & Dui & \\
\hline & $\mathbf{N}$ & $\%$ & $\mathbf{N}$ & $\%$ & $\mathbf{N}$ & $\%$ & $\mathbf{N}$ & $\%$ & \\
\hline Total & 238 & 63,3 & 138 & 36,7 & 299 & 79,5 & 77 & 20,5 & 376 \\
\hline $\begin{array}{l}\text { Activité } \\
\text { Divers } \\
\text { Imprimeurs } \\
\text { Prothèses }\end{array}$ & $\begin{array}{l}79 \\
74 \\
85\end{array}$ & $\begin{array}{l}68,1 \\
53,2 \\
70,2\end{array}$ & $\begin{array}{l}37 \\
65 \\
36\end{array}$ & $\begin{array}{l}31,9 \\
46,8 \\
29,8\end{array}$ & $\begin{array}{r}96 \\
113 \\
90\end{array}$ & $\begin{array}{l}82,8 \\
81,3 \\
74,4\end{array}$ & $\begin{array}{l}20 \\
26 \\
31\end{array}$ & $\begin{array}{l}17,2 \\
18,7 \\
25,6\end{array}$ & $\begin{array}{l}116 \\
139 \\
121\end{array}$ \\
\hline $\begin{array}{l}\text { Taille } \\
1 \text { à } 6 \\
7 \text { à } 10 \\
11 \text { à } 20 \\
\text { Plus de } 20\end{array}$ & $\begin{array}{l}72 \\
90 \\
47 \\
29\end{array}$ & $\begin{array}{l}74,2 \\
67,7 \\
58,8 \\
43,9\end{array}$ & $\begin{array}{l}25 \\
43 \\
33 \\
37\end{array}$ & $\begin{array}{l}25,8 \\
32,3 \\
41,3 \\
56,1\end{array}$ & $\begin{array}{r}81 \\
104 \\
63 \\
51\end{array}$ & $\begin{array}{l}83,5 \\
78,2 \\
78,8 \\
77,3\end{array}$ & $\begin{array}{l}16 \\
29 \\
17 \\
15\end{array}$ & $\begin{array}{l}16,5 \\
21,8 \\
21,3 \\
22,7\end{array}$ & $\begin{array}{r}97 \\
133 \\
80 \\
66\end{array}$ \\
\hline $\begin{array}{l}\text { Opinions } \\
\text { Passifs } \\
\text { Offensifs } \\
\text { Défensifs } \\
\text { Esprit PME } \\
\text { Modernes } \\
\text { Protégés }\end{array}$ & $\begin{array}{l}48 \\
41 \\
25 \\
32 \\
33 \\
59\end{array}$ & $\begin{array}{l}60,8 \\
52,6 \\
69,4 \\
57,1 \\
73,3 \\
72\end{array}$ & $\begin{array}{l}31 \\
37 \\
11 \\
24 \\
12 \\
23\end{array}$ & $\begin{array}{l}39,2 \\
47,4 \\
30,6 \\
42,9 \\
26,7 \\
28\end{array}$ & $\begin{array}{l}60 \\
61 \\
27 \\
45 \\
39 \\
67\end{array}$ & $\begin{array}{l}75,9 \\
78,2 \\
75 \\
80,4 \\
86,7 \\
81,7\end{array}$ & $\begin{array}{r}19 \\
17 \\
9 \\
11 \\
6 \\
15\end{array}$ & $\begin{array}{l}24,1 \\
21,8 \\
25 \\
19,6 \\
13,3 \\
18,3\end{array}$ & $\begin{array}{l}79 \\
78 \\
36 \\
56 \\
45 \\
82\end{array}$ \\
\hline
\end{tabular}

Source : Enquête IRG. 


\subsection{Les conséquences}

Il est clair que les outils commerciaux de la TPE découleront de la conception que se font les dirigeants du commercial en général et de la façon dont ils envisagent l'insertion de leur entreprise dans l'environnement.

Si les outils proprement mercatiques ne sont pas usités, on peut penser que ceux qui fondent, on pourrait dire de façon minimale, la démarche commerciale le soient un peu plus. Mais, même ici, les résultats sont peu convaincants. Près des deux tiers des entreprises de l'échantillon ne disposent pas de plaquette de présentation et près de $80 \%$ d'entre elles n'ont pas réalisé récemment de publipostage ; $62 \%$ des TPE déclarent gérer leur fichier client sur ordinateur (tableau 8), mais ce score ne doit pas faire illusion, $70 \%$ de ces entreprises n'ayant pas de fichier de prospection ( $80 \%$ pour les moins de six salariés). À cet égard, on peut considérer ces chiffres comme révélateurs des véritables préoccupations des dirigeants des TPE où la préférence est nettement donnée à la clientèle existante plutôt qu'à une éventuelle clientèle à conquérir. De ce fait, il y a cohérence entre les opinions de ces dirigeants et leurs pratiques commerciales.

\section{TABLEAU 8}

Gestion des fichiers

\begin{tabular}{|c|c|c|c|c|c|c|c|c|c|}
\hline & \multicolumn{4}{|c|}{ Fichier géré sur ordinateur? } & \multicolumn{4}{|c|}{ Fichier de prospection? } & \multirow{3}{*}{$\begin{array}{c}\text { Tota } \\
\mathbf{N}\end{array}$} \\
\hline & \multicolumn{2}{|c|}{ Non } & \multicolumn{2}{|c|}{ Oui } & \multicolumn{2}{|c|}{ Non } & \multicolumn{2}{|c|}{ Oui } & \\
\hline & $\mathbf{N}$ & $\%$ & $\mathbf{N}$ & $\%$ & $\mathbf{N}$ & $\%$ & $\mathbf{N}$ & $\%$ & \\
\hline Total & 143 & 38,1 & 232 & 61,9 & 259 & 69,1 & 116 & 30,9 & 375 \\
\hline $\begin{array}{l}\text { Activité } \\
\text { Divers } \\
\text { Imprimeurs } \\
\text { Prothèses }\end{array}$ & $\begin{array}{l}55 \\
57 \\
31\end{array}$ & $\begin{array}{l}47,4 \\
41,3 \\
25,6\end{array}$ & $\begin{array}{l}61 \\
81 \\
90\end{array}$ & $\begin{array}{l}52,6 \\
58,7 \\
74,4\end{array}$ & $\begin{array}{l}86 \\
87 \\
86\end{array}$ & $\begin{array}{l}74,1 \\
63,0 \\
71,1\end{array}$ & $\begin{array}{l}30 \\
51 \\
35\end{array}$ & $\begin{array}{l}25,9 \\
37,0 \\
28,9\end{array}$ & $\begin{array}{l}116 \\
138 \\
121\end{array}$ \\
\hline $\begin{array}{l}\text { Taille } \\
1 \text { à } 6 \\
7 \text { à } 10 \\
11 \text { à } 20 \\
\text { Plus de } 20\end{array}$ & $\begin{array}{l}53 \\
54 \\
19 \\
17\end{array}$ & $\begin{array}{l}54,6 \\
40,9 \\
23,8 \\
25,8\end{array}$ & $\begin{array}{l}44 \\
78 \\
61 \\
49\end{array}$ & $\begin{array}{l}45,4 \\
59,1 \\
76,3 \\
74,2\end{array}$ & $\begin{array}{l}79 \\
95 \\
50 \\
35\end{array}$ & $\begin{array}{l}81,4 \\
72,0 \\
62,5 \\
53,0\end{array}$ & $\begin{array}{l}18 \\
37 \\
30 \\
31\end{array}$ & $\begin{array}{l}18,6 \\
28,0 \\
37,5 \\
47,0\end{array}$ & $\begin{array}{r}97 \\
132 \\
80 \\
66\end{array}$ \\
\hline $\begin{array}{l}\text { Opinions } \\
\text { Passifs } \\
\text { Offensifs } \\
\text { Défensifs } \\
\text { Esprit PME } \\
\text { Modernes } \\
\text { Protégés }\end{array}$ & $\begin{array}{l}35 \\
25 \\
11 \\
19 \\
19 \\
34\end{array}$ & $\begin{array}{l}44,3 \\
32,1 \\
30,6 \\
33,9 \\
42,2 \\
42\end{array}$ & $\begin{array}{l}44 \\
53 \\
25 \\
37 \\
26 \\
47\end{array}$ & $\begin{array}{l}55,7 \\
67,9 \\
69,4 \\
66,1 \\
57,8 \\
58\end{array}$ & $\begin{array}{l}58 \\
43 \\
22 \\
40 \\
32 \\
64\end{array}$ & $\begin{array}{l}73,4 \\
55,1 \\
61,1 \\
71,4 \\
71,1 \\
79\end{array}$ & $\begin{array}{l}21 \\
35 \\
14 \\
16 \\
13 \\
17\end{array}$ & $\begin{array}{l}26,6 \\
44,9 \\
38,9 \\
28,6 \\
28,9 \\
21\end{array}$ & $\begin{array}{l}79 \\
78 \\
36 \\
56 \\
45 \\
81\end{array}$ \\
\hline
\end{tabular}

Source : Enquête IRG. 
Paradoxalement, si la fonction commerciale apparaît comme mal maîtrisée et peu structurée, les dirigeants des TPE déclarent dans leur majorité (54\%) qu'elle occupe une position primordiale (tableau 9). L'effet taille est ici très marqué alors qu'il l'est moins lorsqu'on pose la question aux entreprises de savoir si elles jugent leur force commerciale suffisante. On ne sera dès lors pas très surpris du faible pourcentage d'entreprises qui montrent une disposition favorable au recrutement d'un commercial en général et d'un jeune diplômé en techniques de commercialisation en particulier (moins de $20 \%$ en moyenne). On retrouve ici d'ailleurs une tendance profonde de ces entreprises puisque les résultats de notre précédente enquête sur l'innovation technologique montraient des dispositions analogues avec, peut-être, une défiance encore plus marquée à l'égard des systèmes traditionnels de formation (hors apprentissage). Enfin, la mise en perspective des effets passés du recrutement d'un commercial (tableau 10) résonne de façon dissonante avec les effets attendus du recrutement d'un commercial (tableau 11).

Pour la majorité des TPE dont le chiffre d'affaires est inférieur à deux millions de francs, un tel score est vraisemblablement insuffisant pour justifier la création d'un tel poste. En conséquence, on ne sera pas surpris de constater que la volonté

TABleau 9

Jugement sur la force commerciale

\begin{tabular}{|c|c|c|c|c|c|c|c|c|c|}
\hline & \multicolumn{4}{|c|}{$\begin{array}{l}\text { Force commerciale } \\
\text { suffisante? }\end{array}$} & \multicolumn{4}{|c|}{$\begin{array}{c}\text { Fonction commerciale } \\
\text { primordiale? }\end{array}$} & \multirow{3}{*}{$\begin{array}{c}\text { Total } \\
\mathbf{N}\end{array}$} \\
\hline & \multicolumn{2}{|c|}{ Non } & \multicolumn{2}{|c|}{ Oui } & \multicolumn{2}{|c|}{ Non } & \multicolumn{2}{|c|}{ Oui } & \\
\hline & $\mathbf{N}$ & $\%$ & $\mathbf{N}$ & $\%$ & $\mathbf{N}$ & $\%$ & $\mathbf{N}$ & $\%$ & \\
\hline Total & 185 & 49,5 & 189 & 50,5 & 172 & 46,0 & 202 & 54,0 & 374 \\
\hline \multicolumn{10}{|l|}{ Activité } \\
\hline Divers & 61 & 52,6 & 55 & 47,4 & 44 & 37,9 & 72 & 62,1 & 116 \\
\hline Imprimeurs & 72 & 51,8 & 67 & 48,2 & 56 & 40,3 & 83 & 59,7 & 139 \\
\hline Prothèses & 52 & 43,7 & 67 & 56,3 & 72 & 60,5 & 47 & 39,5 & 119 \\
\hline \multicolumn{10}{|l|}{ Taille } \\
\hline 1 à 6 & 53 & 54,6 & 44 & 45,4 & 55 & 56,7 & 42 & 43,3 & 97 \\
\hline 7 à 10 & 55 & 42,0 & 76 & 58,0 & 72 & 55,0 & 59 & 45,0 & 131 \\
\hline 11 à 20 & 48 & 60,0 & 32 & 40,0 & 32 & 40,0 & 48 & 60,0 & 80 \\
\hline Plus de 20 & 29 & 43,9 & 37 & 56,1 & 13 & 19,7 & 53 & 80,3 & 66 \\
\hline \multicolumn{10}{|l|}{ Opinions } \\
\hline Passifs & 45 & 57 & 34 & 43 & 34 & 43 & 45 & 57 & 79 \\
\hline Offensifs & 35 & 44,9 & 43 & 55,1 & 28 & 35,9 & 50 & 64,1 & 78 \\
\hline Défensifs & 22 & 61,1 & 14 & 38,9 & 22 & 61,1 & 14 & 38,9 & 36 \\
\hline Esprit PME & 16 & 28,6 & 40 & 71,4 & 19 & 33,9 & 37 & 66,1 & 56 \\
\hline Modernes & 28 & 62,2 & 17 & 37,8 & 19 & 42,2 & 26 & 57,8 & 45 \\
\hline Protégés & 39 & 48,8 & 41 & 51,3 & 50 & 62,5 & 30 & 37,5 & 80 \\
\hline
\end{tabular}

Source : Enquête IRG. 
TABLEAU 10

Effet du recrutement passé

\begin{tabular}{lcc}
\hline & $\begin{array}{c}\text { Entreprises qui ont recruté } \\
\text { un commercial }\end{array}$ & $\begin{array}{c}\text { Évolution moyenne } \\
\text { du chiffre d'affaires }\end{array}$ \\
\hline Total & 76 & $+51,57$ \\
\hline Activité & 25 & $+52,92$ \\
Divers & 32 & $+48,94$ \\
Imprimeurs & 19 & $+54,21$ \\
Prothèses & & \\
\hline Taille & 8 & $+30,63$ \\
1 à 6 & 20 & $+51,90$ \\
7 à 10 & 24 & $+51,71$ \\
11 à 20 & 24 & $+58,13$ \\
Plus de 20 & & \\
\hline
\end{tabular}

Source : Enquête IRG.

TABleau 11

Effet attendu du recrutement d'un diplômé

\begin{tabular}{lrrrr} 
& NON & OUI & $\%$ & $\begin{array}{c}\text { Moyenne de } \\
\text { progression du chiffre } \\
\text { d'affaires attendu }\end{array}$ \\
\hline Pensé à recruter un jeune diplômé ? & 297 & 12 & 0,04 & 23,75 \\
NON & 36 & 27 & 0,43 & 21,56 \\
OUI & & & & \\
\hline En techniques de commercialisation ? & 326 & 1 & 0,00 & 30 \\
NON & 7 & 38 & 0,84 & 22,03 \\
OUI & & & & \\
\hline
\end{tabular}

Source: Enquête IRG.

de développement affichée des TPE (plus de $80 \%$ des entreprises souhaitent voir progresser leur chiffre d'affaires) ne rime que rarement avec l'idée de développement de la fonction commerciale.

\subsection{L'homogénéité comportementale des TPE}

Nous n'avons pas abordé, jusqu'ici, le problème des profils entrepreneuriaux, au centre de nombre d'études sur la PME (Torrès, 1998). Ce recours aux typologies de type profil avait pour ambition d'expliquer les différences de comportement de ces entreprises. 
En partant du postulat que le rôle du dirigeant était la variable majeure à prendre en considération dans les PME (Candau, 1981), on en est venus fort logiquement à établir des typologies d'entrepreneur censées expliquer les différences constatées. Le problème, c'est qu'en procédant ainsi on en est venus, tout aussi logiquement, à évacuer des dimensions qui pouvaient se révéler tout aussi explicatives et du même coup les «bonnes raisons » (Boudon, 1990) qui poussent les dirigeants de ces entreprises à ne pas adopter une démarche marketing ou pour le moins commerciale. Ainsi, il est certain que la dichotomisation qu'entraîne ce type d'analyse favorise des solutions prescriptives et des jugements comme ceux de Mendelssohn (1991) pour qui la réussite de la petite entreprise passe obligatoirement par l'adoption d'une démarche marketing. L'offre de formation en gestion pour les dirigeants des TPE, qu'elle soit de nature consulaire ou autre, repose grandement sur cette vision des choses et oppose souvent un « idéal-type » managérial à la TPE traditionnelle, donc dépassée. Dans tous les cas, l'adaptation au marché devient une obligation et la seule voie à suivre.

A contrario, ce qu'il faudrait pouvoir expliquer, c'est comment des entreprises qui ne développent aucune démarche commerciale perdurent et peuvent prospérer, même dans des environnements marqués par la concurrence. Dans une perspective plus large, cela devrait conduire à une réflexion sur les conditions de réussite de ces entreprises, sachant que l'on ne pourrait pas faire l'économie des contextes dans lesquels elles se développent.

Le caractère contingent de ces réussites ne doit pas occulter la permanence du fait artisanal en France et dans d'autres pays comme l'Italie. Or, comme l'a montré avec force Harrison (1995), les environnements sont aujourd'hui loin d'être aussi favorables aux petites entreprises que ne l'ont affirmé certains auteurs comme Piore et Sabel (1984). Le paradigme culturel ne doit pas faire oublier la considération de dimensions plus objectives comme celles touchant aux phénomènes de dépendance.

Enfin, on peut se demander si, au fond, on n'aboutit pas, avec une telle grille d'analyse, à une vision déterministe de la réalité. Du point de vue méthodologique, il importe de bien montrer les différences comportementales induites par ces profils. De fait, ce travail a été effectué ( $c f$. section 1.2.). L'important était bien entendu de savoir si ces entrepreneurs avaient des comportements commerciaux différenciés; les analyses de variance réalisées ne permettent pas de conclure dans ce sens, que cela concerne la concentration ou la régularité de la clientèle. On remarque, au contraire, une grande homogénéité dans les résultats, quel que soit le profil de l'entrepreneur. Ainsi, l'importance accordée à la fonction commerciale varie très sensiblement selon le profil. On remarque de la sorte que les chefs d'entreprise appartenant aux catégories « esprit PME » et « modernes », à la question « fonction commerciale primordiale », obtiennent des scores partout supérieurs à ceux des 
chefs d'entreprise appartenant aux catégories « passifs », « protégés » et « défensifs ». Toutefois, sur le plan de l'utilisation des outils (plaquettes, publipostages, fichiers de prospection), on ne note pas de différences significatives.

Ces résultats montrent à l'évidence des appréciations divergentes quant au souhaitable, mais sur le possible, c'est-à-dire leur comportement réel, les différences s'estompent ; ce qui atténue la pertinence des typologies d'entrepreneurs proposés (Woo, Cooper et Dunkelberg, 1991). Si l'on note de la part des premières, et au vu des résultats de l'enquête, une plus grande capacité à utiliser les services des organismes institutionnels de même qu'une attention plus affirmée pour tout ce qui concerne la visibilité externe de leur entreprise, pour autant ces différences n'entraînent pas des trajectoires comportementales contrastées et c'est, en dernier lieu, ce qui nous paraît important de souligner.

L'ouverture sur l'extérieur ne signifie pas ouverture sur le marché ou comportement marketing accentué ; l'ouverture se situant plutôt dans la capacité de l'entreprise à mobiliser des ressources liées à l'information pour répondre aux nouvelles exigences des clients. Dès lors, il devient nécessaire lorsqu'on examine les profils des dirigeants de TPE, d'établir une distinction nette entre ce qui concerne, d'une part, le rapport à l'entreprise et, d'autre part, le rapport à l'environnement. C'est seulement en fonction du second que s'opère la différenciation des comportements, mais, globalement, le rapport à l'entreprise reste le même. La TPE innovante ou marketing ne constituant pas, loin s'en faut, une PME en devenir.

L'existence de profils différenciés pour ce qui concerne le rapport au commercial en général et au marketing en particulier ne doit pas néanmoins, être, négligée ni même sous-estimée. C'est sans doute à partir de ces appréhensions différentes que s'opéreront les évolutions de ces entreprises et, plus profondément, les changements de culture d'entreprise susceptibles de modifier la relation avec l'environnement (Minguzzi et Passaro, 1995). D'un point de vue plus pratique, les stratégies de « satisfaction » qui réussiront seront aussi celles qui s'appuieront sur une connaissance intime du client, pas simplement perçue, et cette connaissance accrue passe par le renforcement de la capacité commerciale, la seule relation technique apparaissant comme insuffisante.

Un rapport d'études, réalisé par des animateurs économiques des métiers et concernant la sensibilisation au marché des entreprises de ce secteur (ISM, 1998), met en lumière le fait que cette sensibilisation réussit d'autant mieux et modifie la nature des outils mis en œuvre que les entrepreneurs sont déjà convaincus de la nécessité de renforcer leur capacité commerciale. Ce renforcement est indissociable, précise le rapport, de la part de ces entreprises, d'une réflexion sur les bases de leur avantage concurrentiel et donc sur une réflexion plus objective où l'offre de l'entreprise est comparée à celle des autres offres existant sur tel ou tel espace concurrentiel. Mais le préalable à cette évolution, souligne une autre étude (Deret, 1999), demeure 
la capacité de l'entrepreneur d'être à l'écoute de ses clients ; c'est à partir de là que sa compétence centrale, son cœur de métier, surtout défini en termes techniques, s'ouvrira aux dimensions plus spécifiquement de gestion (comptable, commercial, organisation). De la sorte, une sensibilisation plus affirmée des besoins du client et de la nécessité de s'y adapter font évoluer la nature du cœur de métier de l'entrepreneur et de la perception qu'il peut en avoir.

\section{Conclusion}

La notion de marché apparaît, du point de vue des TPE, comme une notion ambiguë. Le problème est que la sensibilité au marché est devenue un critère de normalité. C'est cette vision qu'il faut revoir et admettre que, pour beaucoup d'entreprises, l'adaptation n'aurait pas, loin de là, que des conséquences positives. Au-delà des particularités habituellement évoquées lorsqu'on traite de la PME (Torrès, 1998), le mode de relation à l'environnement apparaît comme une caractéristique majeure des TPE qui les distingue à la fois des moyennes et des grandes entreprises. C'est dans cette relation particulière à l'environnement qu'il faut plus sûrement trouver les raisons du désintérêt à l'égard de la démarche commerciale et, plus globalement, du marketing.

\section{Bibliographie}

AMENDOlA, M. et J.L. GAFFARD (1988), La dynamique économique de l'innovation, Paris, Economica.

ATAMER, R. et R. CAlORI (1993), Diagnostic et décisions stratégiques, Paris, Dunod.

Auvolat, M., J.C. LAVIGNe et A. MAYERE (1985), L'artisanat en France; réflexion générale et prospective, Paris, La Documentation française.

Boudon, R. (1990), L'art de se persuader, Paris, Fayard.

CARland, J., F. HoY, W. Boulton et J.A. CARLAND (1984), «Differenciating entrepreneurs from small business owners : a conceptualization », Academy of Management Review, vol. 9, p. 354-359.

CANDAU, P. (1981), «Pour une taxonomie de l'hypofirme », Revue d'économie industrielle, vol. 16, p. 16-33.

DAVID, M. (1998), « Brève histoire de l'artisanat », Cahier ISM.

DAY, G. (1994), «Continuous learning about markets », California Management Review, $n^{\circ} 4$, p. 9-31.

DERET, E. (1999), «Produire pour un marché, quelles compétences ? , Artisans et stratégie commerciale, Cahier ISM.

DICKSON, R.D. (1992), «Toward a general theory of competitive rationality », Journal of Marketing, p. 69-83. 
Dodgson, M. (1996), «Learning trust and interfirm technological linkages : some theoretical associations », dans R. Coombs, A. Richards, P.P. Saviotti et V. Walsh (dir.), Technological Collaboration, Londres, Edgar Edward Publishing, p. 54-75.

Dosi, G. (1984), Technical Change and Industrial Transformation, Londres, MacMillan.

DuCHENEAUT, B. (1995), Enquête sur les PME françaises, identités, contextes, chiffres, Paris, Maxima.

GREPME (1994), Les PME. Bilan et perspectives, ouvrage collectif sous la direction de P.-A. Julien, Paris, Economica.

GirARD, B. (1998), « Pourquoi les PME restent-elles PME ?», Problèmes économiques 2551, p. 11-17.

GiroD, M. (1995), «La mémoire organisationnelle », Revue Française de Gestion, septembre-octobre, p. 30-42.

HARRISON, B. (1995), "Symposium on Harrison's "lean and mean"; what are the questions ?», Small Business Economics, vol. 7, $\mathrm{n}^{\circ} 5$.

HERRMANN, J.L. (1998), « Le marketing en PME manufacturières, les pratiques de quelques entreprises lorraines lors du développement du produit », dans les Actes du IV Congrès francophone sur la PME, du 22 au 24 octobre, Nancy, Metz.

HILLS, G.E. (1987), « Marketing and entrepreneurship research issues : scholarly justification », Proceedings of Research Symposium on the Marketing Entrepreneurship Interface, Chicago, p. 3-15.

ISM (1998), « Animation économique et artisanat », Cahier ISM.

KAMINSKI, P. (1993), «L'innovation dans la micro-entreprise, au cœur de la relation de sous-traitance », SESSI, 41, septembre.

Kotler, P., G.H.G. MACDougall et J.L. PiCARD (1983), Principes de marketing, Québec, Éditions Préfontaine.

LETOWSKI, A. (1987), Les systèmes socio-culturels de l'artisanat face aux mutations, thèse de science des organisations, Université Paris-Dauphine.

MacCarthy, J.E. et W.D PerReault (1984), Basic Marketing, Homewood, Ill., Irwin.

MARCHESNAY, M. (1988), «La mercatique de la petite entreprise », Revue internationale $P M E$, vol. 1 , nos $3-4$, p. 259-276.

MARCheSNAY, M. (1992), «La PME : une gestion spécifique », Problèmes économiques, 20 mai, $\mathrm{n}^{\circ} 2276$.

MARCHESNAY, M. (1994), «Le management stratégique », dans GREPME, Les PME. Bilan et perspectives, Paris, Economica, p. 133-162.

MÉdUS, J.L. et J.-C. PACITTO (1994), «L'innovation technologique dans la très petite entreprise : un premier bilan », Cahier de recherche IRG, $\mathrm{n}^{\circ}$ 94-11.

MENDELSSOHN, J. (1991), «Small firm and the marketing mission », Accountancy, $\mathrm{n}^{\circ} 107$.

MiNGUZZI, A. et R. PASSARO (1995), «L'apprentissage comme facteur d'innovation des entreprises; enquête et exploration dans les petites entreprises italiennes », Actes du congrès «Innovation et organisation des PME», Paris. 
PACITTO, J.C. (1996), «La très petite entreprise innovante : entre réalités et fictions », Cahier de recherche IRG, $\mathrm{n}^{\circ}$ 96-12.

PERREAULT, J.D. (1994), «Le marketing de la PME», dans Les PME. Bilan et perspectives, GREPME, Paris, Economica, p. 163-188.

PIORE, M.J. et C.F. SABEL (1984), The Second Industrial Divide : Possibilities for Prosperity, New York, Basic Books.

PITCHER, P. (1996), Artistes, artisans et technocrates, Paris, Village mondial.

PolanyI, M. (1966), The Tacit Dimension, Londres, Routledge and Kegan Paul.

Polge, M. (1996), L'avantage concurrentiel en petite entreprise, thèse de doctorat NR, Université de Montpellier I, janvier.

PORTER, M.E. (1997), « Plaidoyer pour un retour de la stratégie », L'Expansion Management Review, mars.

REIX, R. (1995), «Savoir tacite et savoir formalisé dans l'entreprise », Revue Française de Gestion, $\mathrm{n}^{\circ} 105$, p. 17-28.

SIMÉONI, M. (1998), «Le rôle du savoir-faire dans la firme artisanale », dans Actes du IVe Congrès francophone sur la PME, du 22 au 24 octobre, Nancy, Metz.

SINKULA, J.M. (1994), « Market information processing and organizational theory », Journal of Marketing, p. 35-45.

SMITH, N. et J.B. MINER (1983), « Type of entrepreneur of firm and entrepreneurial motivation : implication for organizational life cycle theory », Strategic Management Journal, vol. 4, $\mathrm{n}^{\circ} 4$.

STASCH, S.F. et J.L.WARD (1987), « Some observations and research opportunities regarding marketing of smaller business », Proceedings of Research Symposium on the Marketing Entrepreneurship Interface, p. 39-53.

TORRÈs, O. (1998), PME, de nouvelles approches, Paris, Economica.

Woo, C.Y., A.C. Cooper et W.E. Dunkenberg (1991), «The development and interpretation of entrepreneurial typologies », Journal of Business Venturing, vol. 6, $\mathrm{n}^{\circ}$ 2, p. 93-111. 